chert. Vielmehr folgt die Selbstbeschreibung der Sorge eines möglichen Ausschlusses durch die Community.

\title{
4.3 ZWISCHENFAZIT
}

\subsubsection{Das Verhältnis zur Soziologie als fragile Identitätskonstruktion der Selbstbeschreibung}

In der Analyse des Verhältnisses von Soziolog(inn)en zur Soziologie selbst (Abschnitt 4.1) konnten wir sehen, dass es diverse Selbstbeschreibungen auf unterschiedlichen Ebenen gab. Es zeigt sich, dass die Soziologie über keinen konsolidierten Kern verfügt, der ähnliche Selbstbeschreibungen zur Verfügung stellt. Vielmehr zeichnet sich die Soziologie durch eine Interpretationsoffenheit aus, die mit unterschiedlichen Sinnkonstruktionen gefüllt wird. Bezeichnend war zudem, dass keiner der Interviewten äußerte, er habe von Anfang an die Absicht gehabt, Soziologie zu studieren. Selbstbeschreibungen wurden mit diversen biografischen Erfahrungen, Enttäuschungen oder Erwartungen angereichert und die Motivation für Soziologie wurde unterschiedlich begründet. Letztlich konnten zwei immer wiederkehrende Charakteristika der Selbstbeschreibung ausgemacht werden: die Orientierung an epistemisch-kognitiven bzw. an sozial-politischen Kriterien der Sinnkonstruktion. Epistemisch-kognitive Kriterien orientierten sich stärker an einer Selbstbeschreibung der „Soziologie als Wissenschaft“, während eine Ausrichtung an sozial-politischen Kriterien sich an einer „Soziologie als Veränderung der Gesellschaft" orientierte. Bereits im Verhältnis der Interviewten zur Disziplin selbst zeigte sich also, dass die Doppelstruktur der Soziologie, ihre Einbindung in den eigenen Gegenstand, bedeutungsvoll für die Selbstbeschreibungen war. Insbesondere kam dies in den Selbstbeschreibungen zur Frage der Wertneutralität der Soziologie zum Vorschein, und ebenso in der Frage zur Motivation für die Soziologie. In beiden Fällen waren ähnliche Muster der Exklusion vs. Inklusion zu beobachten.

Eine Selbstbeschreibung, die sich an sozial-politischen Kriterien orientierte, war mitunter mit einer Legitimierungsrhetorik verbunden. Ausschlaggebend war dafür, inwieweit sich der oder die Interviewte in einem „Innen“ oder „Außen“ der Disziplin verortete. Die Rolle disziplinärer Referenzfolien gewann in Hinblick auf den Umgang mit Werten in der Soziologie einen besonderen Stellenwert. Auch diese Referenzfolien, beispielsweise disziplinäre Vertreter wie Max Weber oder Theorierichtungen wie die kritische Theorie, dienten zur Legitimie- 
rung des eigenen Standpunkts und wurden teilweise diametral zueinander eingesetzt.

Zudem zeigte sich, dass in der Selbstbeschreibung unterschiedliche Ebenen adressiert wurden. Dominant war einerseits die individuelle, andererseits die disziplinäre Ebene. Hier kam eine Unsicherheit zum Ausdruck, welche Aussagen auf individualisierte Stellungnahmen beschränkt und welche auf disziplinärer Ebene geäußert werden konnten. Die hier dargestellten Selbstbeschreibungen lassen sich als Identitätskonstruktionen ansehen, da sie nicht nur Ausdruck eines Fachdiskurses sind, sondern biografische Aspekte wie auch grundlegende Fragen der Sinngebung, innerer Überzeugungen und der Motivation adressieren. Diese Identitätskonstruktionen legitimieren sich selbst über unterschiedliche rhetorische Strategien, die letztlich den eigenen Standpunkt innerhalb der Community plausibilisieren und rechtfertigen sollen. Als fragil sind diese Konstruktionen deshalb zu bezeichnen, da keine Konstruktion übergreifend für alle Interviewten identifiziert werden konnte und die Interviewten oft auf die individuelle Ebene rekurrierten, um ihren Standpunkt deutlich zu machen. Das Verhältnis der Interviewten zur Soziologie selbst war also von weitreichenden und unterschiedlichen Grenzziehungen zum Untersuchungsgegenstand geprägt.

\subsubsection{Das Verhältnis der Soziologie zum Untersuchungsgegenstand: Die Fremdbeschreibung der Gesellschaft}

In Abschnitt 4.2 hingegen wurden Selbstbeschreibungen zum Verhältnis der Soziologie zu ihrem Untersuchungsgegenstand ausgelotet. Die Interviewten fertigten nicht nur Selbstbeschreibungen an, sondern auch - im systemtheoretischen Vokabular verbleibend - Fremdbeschreibungen der Gesellschaft. Grenzkonstruktionen waren also auch davon geprägt, was die Soziologinnen und Soziologen als „Gesellschaft“ bzw. Untersuchungsgegenstand bestimmten. Zwei dominierende Fremdbeschreibungen ließen sich in diesem Zusammenhang feststellen: erstens die Bestimmung eines „Außen“, das die Soziologie in ihrer Autonomie bedroht und gegen das sie sich abschotten muss, beispielsweise durch Nutzenforderungen oder überhöhte Ansprüche an die Disziplin; zweitens die Bestimmung eines „Außen“, zu dessen Rationalisierung die Soziologie beitragen kann, indem sie übergeordnetes Wissen in den gesellschaftlichen Diskurs einbringt. Beide Formen der Fremdbeschreibungen bringen - jede für sich - eine Distinktion der Soziologie zu ihrem Untersuchungsgegenstand mit sich.

Auch in Abschnitt 4.2 konnte die Doppelstruktur der Soziologie ausgemacht werden. So stellte sich in Hinblick auf Risiken im Umgang zwischen der Sozio- 
logie und ihrem Untersuchungsgegenstand die Frage nach der adäquaten Grenzziehung. Risiken wurden einerseits in falscher Parteinahme für den Gegenstand gesehen (mangelnde Distanz), andererseits in dem Verlust des Alltagswissens, das die Soziologie für ihr Bestehen braucht (zu viel Distanz). Interessanterweise waren Grenzdiskurse nicht ausschließlich in Hinblick auf die Abgrenzung zum eigenen Gegenstand Thema. Vielmehr verlagerten sie sich auf interne Grenzdebatten der Soziologie selbst und auf die damit verbundene Gefahr, durch die Fachkollegen ausgeschlossen zu werden. Es dienten also nicht externe Grenzen als Demarkationskriterien der Soziologie zu ihrem Untersuchungsgegenstand, sondern interne Bewertungsmuster.

\subsubsection{Die Selbstbeschreibung und die Fremdbeschreibung der Soziologie}

Daraus lässt sich schließen, dass für die Grenzkonstruktionen der Soziologie zu ihrem Untersuchungsgegenstand diverse Ebenen der Selbst- und Fremdbeschreibung bestimmend waren. Im Gegensatz zu systemtheoretischen Konzeptionierungen von Selbstbeschreibungen, die sich lediglich auf der Ebene des Systems verorten und emergent sind, konnten in der Interviewanalyse zwei Bezugsebenen ausgemacht werden: die individuelle Ebene sowie die institutionelle Ebene. Letztere wurde überwiegend auf der disziplinären Ebene adressiert; die organisationale Ebene spielte eine untergeordnete Rolle. Die Selbstbeschreibungen dienten der Grenzziehung zum Untersuchungsgegenstand, gewannen jedoch auf den Bezugsebenen unterschiedliche Qualität.

Individualisierte Selbstbeschreibungen grenzten sich meist von der disziplinären Ebene ab, etwa mit der rhetorischen Markierung ,aus meiner Sicht“ oder „das ist jetzt meine ganz persönliche Meinung“. Selbstbeschreibungen auf der disziplinären Ebene wurden für die gesamte Disziplin bzw. die Fachkollegenschaft geltend gemacht. Diese Selbstbeschreibungen äußerten sich rhetorisch durch „Aufgabe der Soziologie ist es“ oder „es ist wohl Konsens, dass...“. Auf Ebene der Organisation wurde ein expliziter Praxisbezug entweder über diese selbst vertreten oder auch abgelehnt. Da sie in den Interviews eine untergeordnete Rolle spielte, soll sie in den weiteren Ausführungen zurückgestellt werden.

Individualisierte Selbstbeschreibungen thematisierten oft sozial-politische Motive für das Betreiben der Soziologie, etwa „Aufklärung“, „Einfluss“ oder „Gesellschaftsveränderung“. Auf der disziplinären Ebene wurden Selbstbeschreibungen eher geäußert, um Grenzen gegen externe Einflüsse zu sichern. Die Sorge um eine „mangelnde Autonomie“ der Soziologie gegenüber vereinnahmenden Praxisansprüchen oder Bedenken, dass sich soziologisches Wissen zu 
wenig von anderen Wissensformen abgrenzt, wurde deshalb eher auf der disziplinären Ebene geäußert.

Hier spielt jedoch eine weitere, dritte Ebene in die Grenzkonstruktionen zum Untersuchungsgegenstand hinein: Die (Fremd-)Beschreibung der Gesellschaft. Denn inwieweit diese als Referenz für überhöhte Ansprüche oder als rationalisierungsbedürftiges Konglomerat gesehen wurde, bestimmte letztlich, wie die Grenzen zum Untersuchungsgegenstand gezogen wurden. Daraus ergibt sich eine triadische Struktur der Selbstbeschreibung (siehe Abbildung 4):

\section{Abbildung 4: Triadische Struktur der Selbst- und Fremdbeschreibung} der Soziologie

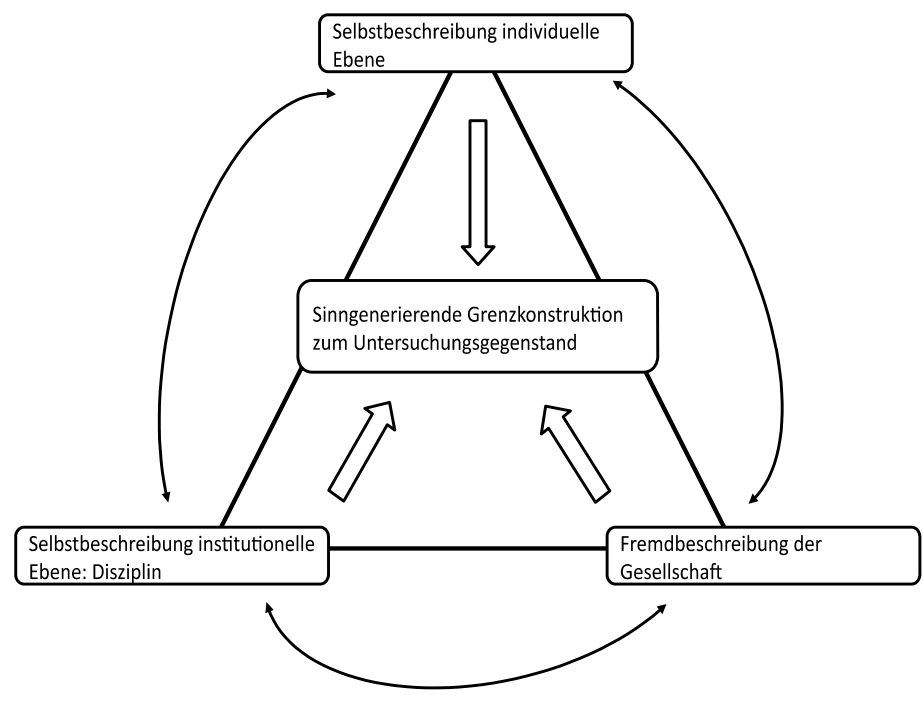

Die sinngenerierenden Grenzkonstruktionen zum Untersuchungsgegenstand setzen sich also aus individuellen Selbstbeschreibungen, disziplinären Selbstbeschreibungen und der Fremdbeschreibung des Untersuchungsgegenstandes zusammen.

Wir haben gesehen, dass sich insbesondere individuelle Selbstbeschreibungen der Soziologie durch eine hohe Interpretationsoffenheit auszeichnen. Sie differierten in Hinblick auf ihre Sinnkonstruktionen und Begründungsmuster und zeichneten sich durch eine Referenzierung einerseits auf epistemisch-kognitive und andererseits auf sozial-politische Kriterien aus. Je nachdem, wie sich der bzw. die Interviewte in der Community situierte, fiel der Einsatz rhetorischer Rechtfertigungsstrategien in der eigenen Selbstbeschreibung aus. Dementspre- 
chend war die Ebene der individuellen Selbstbeschreibung mit der der disziplinären Selbstbeschreibung verbunden. Auch kann ein Zusammenhang zwischen den Selbstbeschreibungen und der Fremdbeschreibung der Gesellschaft ausgemacht werden: Je nachdem, wie Soziologinnen und Soziologen ihr Verhältnis zur Soziologie und ihr Verständnis von einem Auftrag der Soziologie auf individueller und disziplinärer Ebene definierten, fiel auch die Fremdbeschreibung der Gesellschaft aus. 
